\title{
Efficient Shaped-Beam Reflectarray Design Using Machine Learning Techniques
}

\author{
Daniel R. Prado ${ }^{\# 1}$, Jesús A. López-Fernández ${ }^{* 2}$, Manuel Arrebola ${ }^{* 3}$, George Goussetis ${ }^{\# 4}$ \\ ${ }^{\#}$ Institute of Sensors, Signals and Systems, Heriot-Watt University, Edinburgh, U.K. \\ * Group of Signal Theory and Communications, Universidad de Oviedo, Spain \\ $\left\{{ }^{1} \mathrm{dr} 38,{ }^{4}\right.$ g.goussetis $\} @$ hw.ac.uk, $\left\{{ }^{2}\right.$ jelofer, ${ }^{3}$ arrebola $\} @$ uniovi.es
}

\begin{abstract}
This papers introduces the use of machine learning techniques for an efficient design of shaped-beam reflectarrays considerably accelerating the overall process while providing accurate results. The technique is based on the use of Support Vector Machines (SVMs) for the characterization of the reflection coefficient matrix, which provides an efficient way for deriving the scattering parameters associated with the unit cell dimensions. In this way, the SVMs are used within the design process to obtain a reflectarray layout instead of a Full-Wave analysis tool based on Local Periodicity (FW-LP). The accuracy of the SVMs is assessed and the influence of the discretization of the angle of incidence is studied. Finally, a considerable acceleration is achieved with regard to the FW-LP and other works in the literature employing Artificial Neural Networks.
\end{abstract}

Keywords-Machine Learning, Support Vector Machine (SVM), reflectarray

\section{INTRODUCTION}

The efficient analysis and synthesis of reflectarray antennas continues to be a challenging task three decades after the popularization of these antennas [1]-[5]. Although the use of a Full-Wave analysis technique based on Local-Periodicity (FW-LP) has been used with success for the analysis, design [1] and even direct optimization [6] of reflectarray antennas, it is still desirable to achieve faster computations to tackle ever growing problems, specially those dealing with optimization of very large reflectarrays for space applications where the crosspolar pattern needs to be minimized while obtaining a copolar shaped pattern [1], [6]. This has been addressed before with databases [2], but the number of entries grows exponentially with the number of variables. Artificial Neural Networks (ANNs) [3], [4] have also been used. However, limited results have been obtained regarding the characterization of the full reflection coefficient matrix. In addition, ANNs also have the problem of overfitting. A more recent approach consists in the use of Support Vector Machines (SVMs), which is a robust machine learning technique along with some strategies to reduce the dimensionality of the problem to obtain more accurate results [5].

In this paper, SVMs [7] are used for the first time to perform a reflectarray design in order to obtain the dimensions of the elements that match the required phase-shift to obtain a shaped pattern. The paper describes the proposed design process, its speed as well as its accuracy when compared with the design using a FW-LP technique, the Method of Moments (MoM-LP) of [8] in this case. To reduce the number

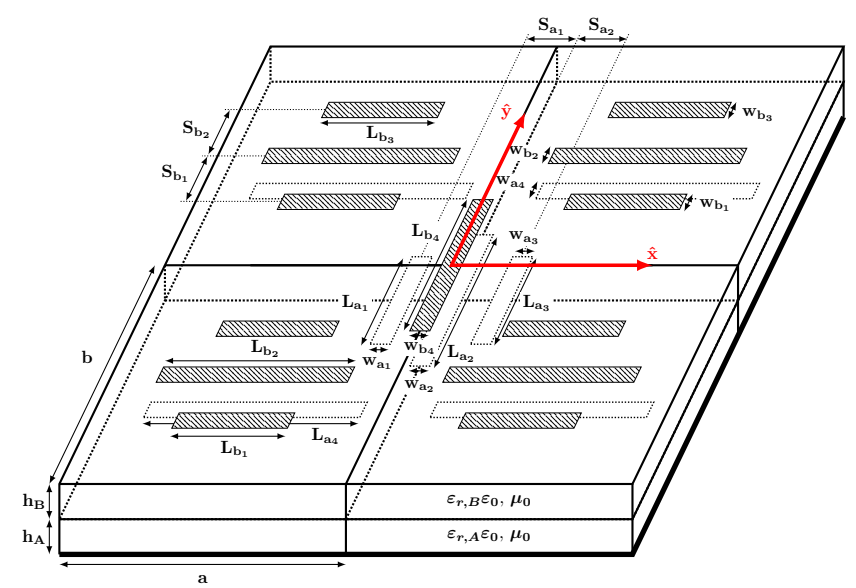

Fig. 1. Reflectarray unit cell based on two sets of four parallel dipoles in two different layers

of SVMs models, the angle of incidence of the wave coming from the feed and impinging at each reflectarray element is discretized. The error of this approximation is assessed and put in perspective with the achieved acceleration and accuracy of the overall process.

\section{Reflectarkay UNit Cell Characterization}

The reflectarray unit cell is the same employed in [5] and shown here in Fig. 1 for convenience. It is comprised of two sets of four parallel dipoles in two different layers. Each set of dipoles controls the phase-shift for each linear polarization. Thus, this unit cell can be employed for the design of dual-polarized reflectarrays. Since the dipoles lengths are the main factor which contribute to the phase-shift produced by this unit cell, the rest of the parameters are fixed to obtain the SVM models. In particular, the working frequency is set to $25.5 \mathrm{GHz}$, the periodicity to $5.84 \mathrm{~mm}$ in both directions, the width of the dipoles to $0.5 \mathrm{~mm}$ and the substrate for the two layers is the CuClad 233 with $\varepsilon_{r}=2.33, \tan \delta=0.0013$ and thickness $0.787 \mathrm{~mm}$.

The goal of the unit cell characterization is to obtain a model of the matrix of reflection coefficients with the SVM. This matrix relates the tangential incident field coming from the feed at each reflectarray element and the tangential reflected field following the equation:

$$
\vec{E}_{\mathrm{ref}}^{X / Y}\left(x_{m}, y_{n}\right)=\mathbf{R}^{m n} \cdot \vec{E}_{\mathrm{inc}}^{X / Y}\left(x_{m}, y_{n}\right),
$$


where the superscript indicates the antenna polarization, $\left(x_{m}, y_{n}\right)$ are the coordinates of the $(m, n)$ th element and

$$
\mathbf{R}^{m n}=\left(\begin{array}{cc}
\rho_{x x}^{m n} & \rho_{x y}^{m n} \\
\rho_{y x}^{m n} & \rho_{y y}^{m n}
\end{array}\right),
$$

is the reflection coefficient matrix. Since the reflection coefficients are complex, a different SVM model will be obtained for the real and imaginary parts following the guidelines in [5]. Only two geometrical variables are taken into account, $T_{x}$ and $T_{y}$, controlling the dipoles oriented in $\hat{x}$ and $\hat{y}$, respectively (see Fig. 1). The rest of the dipoles will be proportional to those values according to the following relations:

$$
\begin{aligned}
& L_{a_{4}}=T_{x} ; L_{b_{1}}=L_{b_{3}}=0.63 T_{x} ; L_{b_{2}}=0.93 T_{x}, \\
& L_{b_{4}}=0.95 T_{y} ; L_{a_{1}}=L_{a_{3}}=0.58 T_{y} ; L_{a_{2}}=T_{y} .
\end{aligned}
$$

In order to further reduce the dimensionality of the problem and increase accuracy, one SVM model will be obtained per angle of incidence. In turn, to reduce the total number of SVMs, the angles of incidence $(\theta, \varphi)$ from the feed to each element will be discretized in steps of $10^{\circ}$. This discretization will introduce an error in the design process which will be assessed in a following section.

\section{REFLECTARRAY DESIGN PROCEDURE}

The goal of the reflectarray design procedure is to compute the dipole lengths that provide the phase-shift at each element necessary to obtain a certain radiation pattern. The required phase-shift is obtained after a synthesis procedure (or analytically for certain canonical radiation patterns) [1]. Since the unit cell shown in Fig. 1 is able to deal with dual-polarized reflectarrays, two phase-shift distributions will be considered, one per polarization. Each group of dipoles will accomplish a different phase-shift.

First, a table of phase-shifts is obtained varying the $T_{x}$ and $T_{y}$ in little steps. The phase-shift obtained modifying each variable is practically uncoupled [9], so this is done independently for each polarization. In this way, we select two values for each variable $T_{x}$ and $T_{y}$ that provide a phase-shift that is close to the required one, but a little above and below the exact value. Then, using a zero-finding routine (Newton-Raphson method, for example), the exact value for both polarizations is found, taking into account the little coupling between the two polarizations that there may exist. Following this approach, $T_{x}$ and $T_{y}$ are found for each reflectarray element, and given the relations in (3), all dipole dimensions are obtained. The last task would be to simulate the reflectarray to obtain the radiation patterns.

This design process is usually done either by employing a commercially available full-wave simulation software, which is very time-consuming, or in-house full-wave analysis tools [1], which are substantially faster but only suitable for certain unit cells. However, the design process involves hundreds or even thousands of calls to this tool, and can take several hours to complete a design for a very large reflectarray [10]. Thus, this
Table 1. Computing time in seconds of the design procedure using MoM-LP and SVM in sequential and parallel implementations of a reflectarray comprised of 900 elements.

\begin{tabular}{ccc}
\hline & Sequential & Parallel \\
\hline MoM-LP & 2492.16 & 390.90 \\
SVM & 2.83 & 0.69 \\
\hline
\end{tabular}

process may be accelerated with the use of equivalent models of the reflectarray cell based on SVMs.

\section{RESULTS}

\section{A. Antenna Specifications}

The design process described in Section III was applied to a reflectarray with a radiation pattern for central stations of cellular systems, which consists of a squared cosecant pattern in elevation and a sectorial beam in azimuth. The reflectarray is comprised of 900 elements in a regular grid of $30 \times 30$. The frequency, periodicity and unit cell characteristics are the same used for the SVM training in Section II. The feed is placed at $(-94,0,214) \mathrm{mm}$ with regard to the center of the reflectarray. Discretizing the incident angles in steps of $10^{\circ}$ for $\theta$ and $\varphi$ gives 57 combinations of $(\theta, \varphi)$ pairs, which are reduced to 31 by using symmetries.

Three different designs were carried out. One with SVM, and two with the MoM-LP using the real angles of incident for each reflectarray element and another with the same discretization of the angles of incidence as used in the SVM. This way, both the SVM accuracy and the influence of the discretization of the angles of incidence may be assessed independently.

\section{B. Computational Acceleration}

Regarding computing time, the simulations were carried out in a laptop with an Intel Core i7-4712MQ CPU at $2.30 \mathrm{GHz}$ (8 CPU, 4 physical plus 4 virtual using hyperthreading). The design procedure can also be parallelized since adjusting the dimensions of one element is an independent task for each element due to the local periodicity assumption.

Computing time for both sequential and parallel implementations for MoM-LP and SVM is shown in Table 1. As it can be seen, an acceleration factor of 880 is obtained for the design in sequential mode (using only one CPU) and a factor of 566 is obtained when the design is parallelized, processing one reflectarray element per available thread. As comparison, in [10] a reflectarray design using ANNs is reported to have an acceleration factor of 206 in sequential mode. However, a different MoM-LP tool was used in that case. Since in [10] a reflectarray comprised of 5180 elements was designed, which has 5.76 times more elements than the one considered in this work, it is expected to carry out the design of such large reflectarray in less than 17 seconds using the SVM in sequential mode, which is around 5 times faster than the time reported in [10] using ANNs. In addition, even faster computing time is achieved by 
parallelizing the procedure, which is not considered in [10]. The parallelization is very important when carry out direct optimization of reflectarray antennas [6].

\section{SVM Accuracy for Reflectarray Design}

In order to assess the SVM accuracy, the SVM design will be compared with the design with the MoM-LP which employed the same discretization of the angles of incidence as the SVM. The fact that the SVM model does not provide exactly the same reflection coefficient matrix of (2) will mean that the dipole lengths (i.e., $T_{x}$ and $T_{y}$ ) will be slightly different after the design process finishes.

Once the $T_{x}$ and $T_{y}$ dimensions have been obtained, the maximum difference between the SVM and MoM-LP designs were calculated. It is $12 \mu \mathrm{m}$, but the absolute mean deviation is less than $3 \mu \mathrm{m}$ for both variables. In addition, the relative error was also computed, resulting in a maximum relative error of $0.43 \%$ for $T_{x}$ and $0.28 \%$ for $T_{y}$; while the mean relative error is only $0.078 \%$ and $0.062 \%$ for $T_{x}$ and $T_{y}$, respectively. This means that, under the same conditions, the SVM provides enough accuracy to carry out reflectarray design. Indeed, as shown in Fig. 2 the simulation of both designs give radiation patterns which are very similar. In fact, the two-norm error for the radiation pattern in the whole visible region for a grid of $512 \times 512$ points and taking the MoM-LP simulation as reference, is only of $0.007 \%$ for the copolar pattern and $0.98 \%$ for the crosspolar pattern, both in $\mathrm{X}$ polarization. Similar results were obtained for $\mathrm{Y}$ polarization.

\section{Influence of the Discretization of the Angles of Incidence}

Next, by comparing the design obtained with the SVM and the MoM-LP which uses the real angles of incidence, the influence of the discretization of those angles will be addressed. It is noted that the differences in the radiation pattern for the previous case were negligible, because the SVM provides a very accurate model.

Fig. 3(a) shows the value of the $T_{x}$ variables obtained after the design using the SVM. Compared to the design using MoM-LP with the real angles of incidence, now the maximum length difference is $30 \mu \mathrm{m}$ (almost three times larger than the previous case), while the mean absolute deviation is $4.4 \mu \mathrm{m}$ for $T_{x}$ and $4.2 \mu \mathrm{m}$ for $T_{y}$. Fig. 3(b) shows the relative error for the worst case, which is for the $T_{x}$ variables, where the maximum relative error is $1.80 \%$. For $T_{y}$ the maximum relative error is $1.30 \%$. Regarding the mean relative error, it is $0.14 \%$ for both $T_{x}$ and $T_{y}$. As it can be seen, now the differences in lengths and relative error are higher, which is expected since the design conditions regarding the angle of incidence for each element are different.

Finally, Fig. 4 shows the radiation patterns obtained from both designs. There is high degree of agreement in the copolar patterns between the SVM and MoM-LP designs and simulations, although this is not true for the crosspolar pattern, where there are some discrepancies for values below $-20 \mathrm{~dB}$. Nevertheless, the prediction of the maximum values of the crosspolar pattern is still accurate, being the difference in

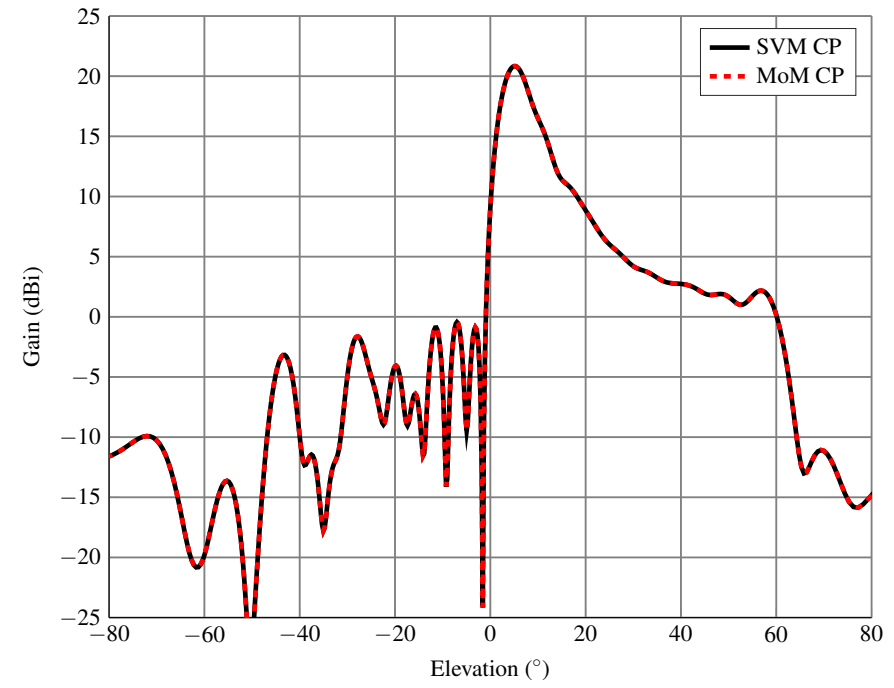

(a)

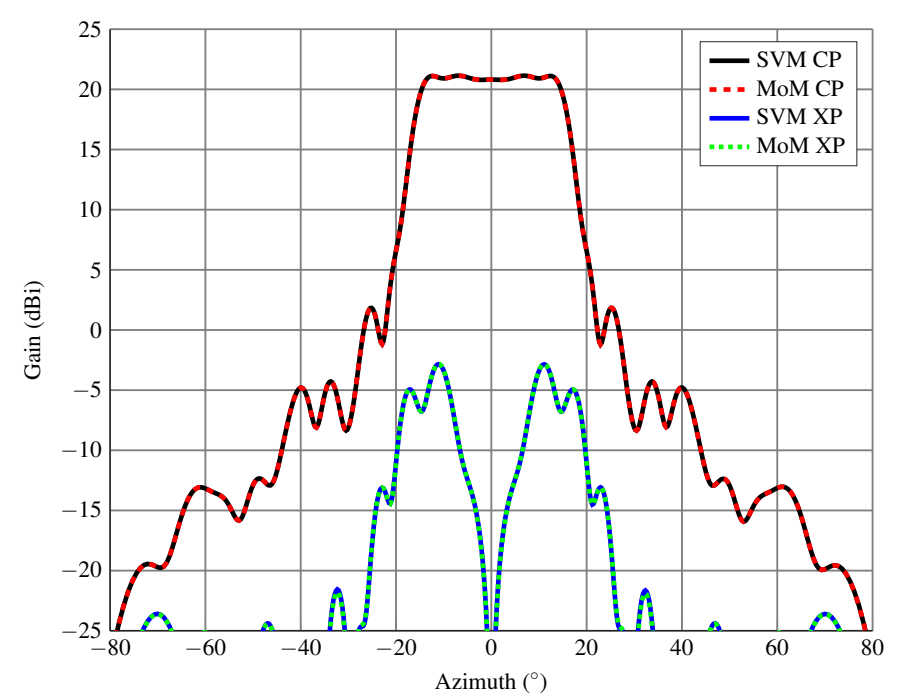

(b)

Fig. 2. Main cuts of the copolar and crosspolar patterns (X polarization) in (a) elevation (azimuth at $0^{\circ}$ ) and (b) azimuth (elevation at $5.4^{\circ}$ ), comparing simulations of the MoM-LP and SVM using the same discretization of angles of incidence.

crosspolar gain of only $0.37 \mathrm{~dB}$. The two-norm error was also calculated in the same conditions as before and it is $0.12 \%$ for the copolar pattern and $12.56 \%$ for the crosspolar pattern. This last error is high since the two-norm error is a relative error and for very low levels of the crosspolar pattern the discrepancies between SVM and MoM-LP are larger.

\section{CONClusion}

Machine learning techniques have been employed for the acceleration of reflectarray antennas design. The accuracy of the technique and the influence of the discretization of the angle of incidence have been assessed for a shaped-beam reflectarray, and a high degree of agreement with MoM-LP design and simulations has been obtained. Some discrepancies for low levels of the crosspolar pattern have been identified, 


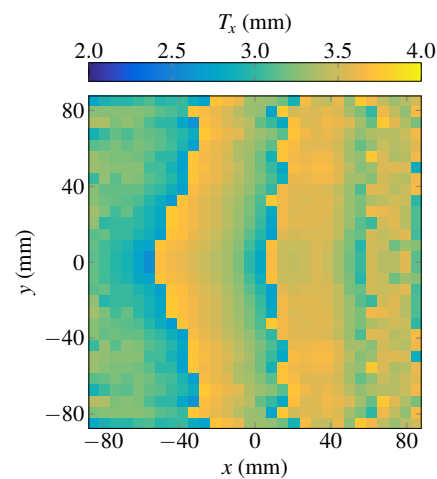

(a)

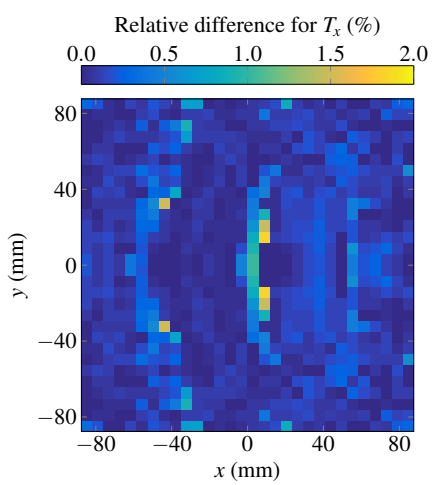

(b)
Fig. 3. For the SVM design: (a) obtained values of $T_{x}$ geometrical variables; (b) relative difference of $T_{x}$ with regard to the MoM-LP design using the real angles of incidence.

but they are produced at very low levels, where errors due to tolerances in the manufacturing and measurement processes may play a more important role. Thus, it has been shown that the use of SVM for reflectarray design is a powerful tool able to accelerate the process between two and three orders of magnitude while providing high accuracy.

\section{ACKNOWLEDGMENT}

This work was supported in part by the European Space Agency (ESA) under contract ESTEC/AO/1-7064/12/NL/MH; by the Ministerio de Economía y Competitividad (Spanish Government), under projects TEC2017-86619-R (ARTEINE) and TEC2016-75103-C2-1-R (MYRADA); and by the Gobierno del Principado de Asturias through Programa "Clarín" de Ayudas Postdoctorales / Marie Courie-Cofund under project ACA17-09.

\section{REFERENCES}

[1] J. Huang and J. A. Encinar, Reflectarray Antennas. Hoboken, NJ, USA: John Wiley \& Sons, 2008.

[2] M. Zhou, S. B. Sørensen, O. S. Kim, E. Jørgensen, P. Meincke, and O. Breinbjerg, "Direct optimization of printed reflectarrays for contoured beam satellite antenna applications," IEEE Trans. Antennas Propag., vol. 61, no. 4, pp. 1995-2004, Apr. 2013.

[3] P. Robustillo, J. Zapata, J. A. Encinar, and J. Rubio, "ANN characterization of multi-layer reflectarray elements for contoured-beam space antennas in the Ku-band," IEEE Trans. Antennas Propag., vol. 60, no. 7, pp. 3205-3214, Jul. 2012.

[4] V. Richard, R. Loison, R. Gillard, H. Legay, and M. Romier, "Loss analysis of a reflectarray cell using ANNs with accurate magnitude prediction," in $11^{\text {th }}$ European Conference on Antennas and Propagation (EuCAP), Paris, France, Mar. 19-24, 2017, pp. 2402-2405.

[5] D. R. Prado, J. A. López-Fernández, G. Barquero, M. Arrebola, and F. Las-Heras, "Fast and accurate modeling of dual-polarized reflectarray unit cells using support vector machines," IEEE Trans. Antennas Propag., vol. 66, no. 3, pp. 1258-1270, Mar. 2018.

[6] D. R. Prado, M. Arrebola, M. R. Pino, R. Florencio, R. R. Boix, J. A. Encinar, and F. Las-Heras, "Efficient crosspolar optimization of shaped-beam dual-polarized reflectarrays using full-wave analysis for the antenna element characterization," IEEE Trans. Antennas Propag., vol. 65, no. 2, pp. 623-635, Feb. 2017.

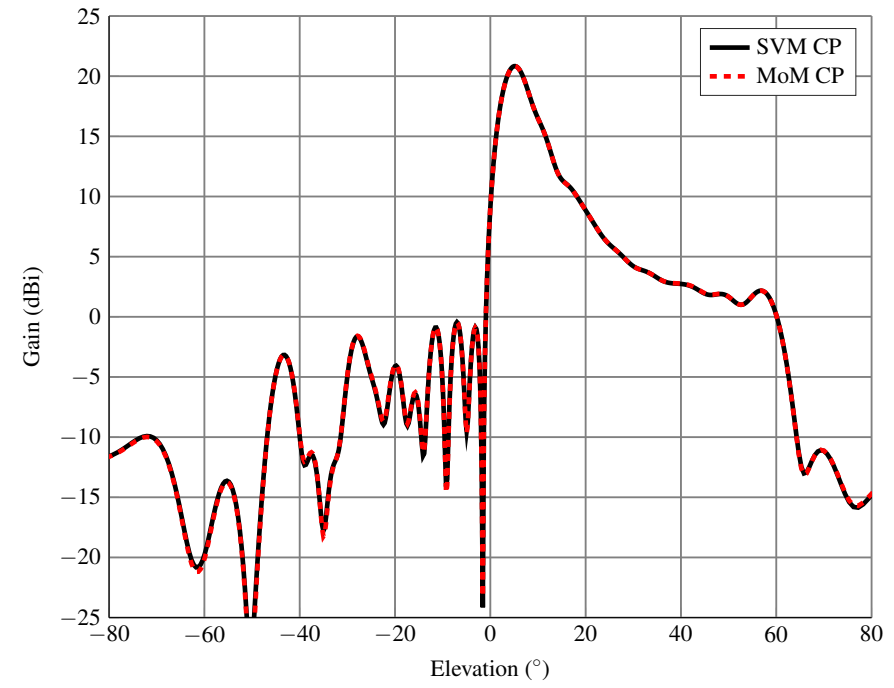

(a)

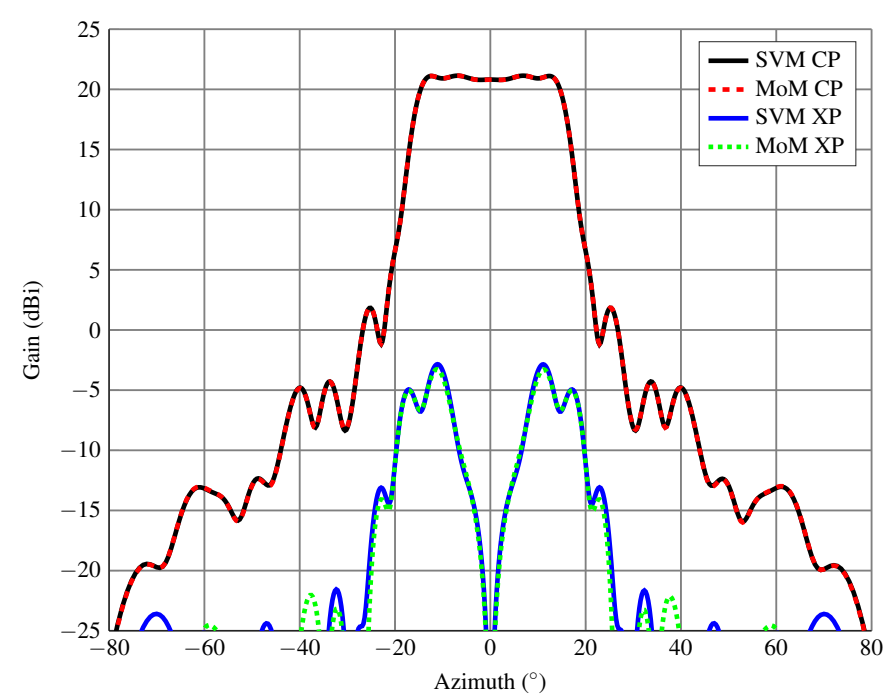

(b)

Fig. 4. Main cuts of the copolar and crosspolar patterns (X polarization) in (a) elevation (azimuth at $0^{\circ}$ ) and (b) azimuth (elevation at $5.4^{\circ}$ ), comparing simulations of the MoM-LP and SVM, where in this cases MoM-LP employs the real angles of incidence at each element to carry out the design.

[7] C.-C. Chang and C.-J. Lin, "LIBSVM: A library for support vector machines," ACM Trans. Intell. Syst. Technol., vol. 2, no. 3, pp. 27:1-27:27, Apr. 2011, software available at http://www.csie.ntu.edu.tw/ cjlin/libsvm.

[8] R. Florencio, R. R. Boix, and J. A. Encinar, "Enhanced MoM analysis of the scattering by periodic strip gratings in multilayered substrates," IEEE Trans. Antennas Propag., vol. 61, no. 10, pp. 5088-5099, Oct. 2013.

[9] R. Florencio, J. A. Encinar, R. R. Boix, V. Losada, and G. Toso, "Reflectarray antennas for dual polarization and broadband telecom satellite applications," IEEE Trans. Antennas Propag., vol. 63, no. 4, pp. 1234-1246, Apr. 2015.

[10] P. Robustillo, J. Zapata, J. A. Encinar, and M. Arrebola, "Design of a contoured-beam reflectarray for a eutelsat european coverage using a stacked-patch element characterized by an artificial neural network," IEEE Antennas Wireless Propag. Lett., vol. 11, pp. 977-980, 2012. 